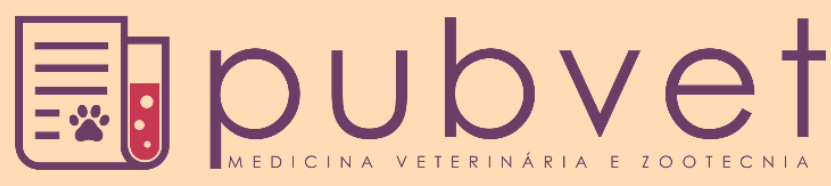

https://doi.org/10.31533/pubvet.v15n03a768.1-6

\title{
Histiocitoma fibroso maligno em plano nasal de um cão: Relato de caso
}

\author{
Nayadjala Távita Alves Santos ${ }^{1 *}{ }^{\bullet}$, Carla Fernanda da Conceição Medeiros ${ }^{2} \bullet$, Lilian Rayanne de

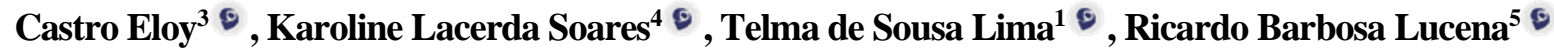 \\ 'Doutoranda pelo Programa de pós-graduação em Medicina Veterinária da Universidade Federal Rural de Pernambuco-UFRPE, \\ Departamento de Medicina Veterinária, Recife-PE, Brasil. \\ ${ }^{2}$ Médica Veterinária, possui Residência em Clínica Médica de Pequenos Animais pelo Programa de Pós-Graduação Lato Sensu da \\ Universidade Federal da Paraíba-UFPB, Departamento de Medicina Veterinária, Areia-PB, Brasil. \\ ${ }^{3}$ Graduanda em Medicina Veterinária pela Universidade Federal da Paraíba-UFPB, Areia-PB, Brasil. \\ ${ }^{4}$ Mestranda pelo Programa de pós-graduação em Ciência Animal da UFPB, Departamento de Medicina Veterinária, Areia-PB, Brasil. \\ ${ }^{5}$ Docente de Medicina Veterinária, Departamento de Medicina Veterinária/Patologia Animal da UFPB, Areia-PB, Brasil. \\ *Autor para correspondência, E-mail: nayadjalat@gmail.com
}

Resumo. Um cão, fêmea, poodle, 8 anos, apresentou queixa principal de lesão ulcerada em plano nasal e epixtase, com evolução de aproximadamente seis meses. O animal foi submetido a anestesia para retirada do nódulo de $0,5 \mathrm{~cm}$ de diâmetro. $\mathrm{O}$ material foi encaminhado para exame histopatológico de rotina com coloração de Hematoxilina-Eosina (HE) e respectivamente imuno-histoquímica. No exame histopatológico notou-se uma massa nodular, com bordos mal definidos, constituída por células arredondadas de citoplasma escasso e cromatina nuclear condensadas que iam desde a derme profunda e dissecavam parte da epiderme. Essas células apresentavam um padrão de pleomorfismo alto e alta relação núcleo:citoplasma. Entremeando o estroma observou-se grande quantidade de estruturas vasculares que vão desde a superfície basal até a derme superficial. O exame imuno-histoquímico revelou forte marcação para o complexo principal de histocompatibilidade MHCII, que cora fortemente linfócitos e histiócitos, que correspondem a estrutura tumoral e proliferativa das células neoplásicas do tumor. Os tumores histiocíticos, bem como histiocitomas são relativamente comuns em cães jovens e mais raramente os histiocitomas fibrosos malignos são diagnosticados em cães, ocorrendo mais comumente em humanos. Nesse relato são descritas as características patológicas e imuno-histoquímicas que permitiram o diagnóstico, possibilitando a diferenciação entre histiocitomas não fibróticos e outras neoplasias caninas comuns.

Palavras chave: Dermatofibroma, dermatopatologia, imuno-histoquímica

\section{Malignant fibrous histiocytoma in the nasal plano f a dog: Case report}

Abstract. One dog, female, poodle, 8 years old, presented the main complaint of ulcerated lesion in the nasal plane, with evolution of approximately six months. The animal was submitted to anesthesia to remove the $0.5 \mathrm{~cm}$ diameter nodule. The material was sent for routine histopathological examination with Hematoxylin-Eosin (HE) staining and immunohistochemistry, respectively. Histopathological examination showed a nodular mass, with ill-defined edges, consisting of rounded cells of sparse cytoplasm and condensed nuclear chromatin that extended from the superficial dermis and dissected part of the epidermis. These cells had a high pleomorphism pattern and a high nucleus: cytoplasm ratio. Intermingling the stroma, a large number of vascular structures were observed, ranging from the basal surface to the superficial dermis. Immunohistochemical examination revealed strong marking for the main MHCII histocompatibility complex that strongly stains lymphocytes and histiocytes that correspond to the tumoral and proliferative structure of the tumor's neoplastic cells. Histiocytic tumors as well as histiocytomas are 
relatively common in young dogs and fibrous histiocytomas are more rarely diagnosed in dogs, occurring more commonly in humans. In this report, the pathological and immunohistochemical characteristics that allowed the diagnosis are described, enabling the differentiation between non-fibrotic histiocytomas and other common canine neoplasms.

Keywords: Dermatofibroma, dermatopathology, immunohistochemistry

\section{Introdução}

Os histiócitos são subconjuntos de leucócitos, e os tumores histiocíticos são neoplasmas resultantes da proliferação dessas células, consideradas neoplasias comuns em cães jovens, podendo ser benignos ou malignos. Podem apresentar-se com diversos padrões e em diversas localidades sendo mais comuns em membros ou extremidades distais, bem como em região de face e pinas. Dividem-se em histiocitoma cutâneo, sarcoma histiocítico, nódulo fibroistiocítico reativo, histiocitose das células de langerhans e histiciocitose progressiva das células dendríticas (Affolter \& Moore, 2006; Gross et al., 2008; Pinto da Cunha et al., 2014).

O dermatofibroma ou histiocitoma fibroso é uma condição dermatológica pouco comum em cães e apresenta diversos padrões histológicos, podendo ser classificado como benigno ou maligno de acordo com esses padrões. Por conseguinte, deve ser diferenciado de outros tumores malignos de células redondas, bem como das demais histiocitoses e histiocitomas (Doyle \& Fletcher, 2013). É definida como uma alteração da reação de células histiocíticas, que consistem em linfócitos diferenciados, células inflamatórias e vasos sanguíneos. No plano nasal esses tumores são mais raros, apresentando-se mais comumente, como já relatado em região de membros.

A classificação histopatológica dos histiocitomas fibrosos em cães consiste em subtipos que vão variar de acordo com o padrão e predominância dos tipos de células, são eles os histiocitomas fibrosos estoriforme-pleomórficos, de células gigantes, inflamatório e mixóide (Goldschmidt \& Hendrick, 2002; Goldschmidt \& Goldschmidt, 2017; Meuten, 2016). Deve ser diferenciado de outros neoplasmas cutâneos como os mastocitomas e sarcomas, os histiocitomas fibrosos bem diferenciados podem ser distinguidos de neoplasias de origem vascular como hemangiomas e hamartomas.

O diagnóstico do histiocitoma é feito pela técnica de imuno-histoquímico, que pode ainda auxiliar na classificação do subtipo histológico dos histiocitomas fibrosos (Stilwell \& Rissi, 2018). Esta técnica tem também relevância clínica, pois fornece apoio no prognóstico e tratamento do animal. Podem ser aplicados anticorpos que marcam células histiocíticas, como CD45, CD18, CD1, MHC II (major histocompatibility complex) que marcam também células de origem histiocítica (Affolter \& Moore, 2006; Gross et al., 2008; Pinto da Cunha et al., 2014).

Nesse relato são descritas as características patológicas e imuno-histoquímicas que permitiram o diagnóstico, possibilitando a diferenciação entre histiocitomas não fibróticos e outras neoplasias caninas oriundas de histiócitos, bem como os tumores cutâneos igualmente comuns como mastocitomas e sarcomas, que são os principais diferenciais dos tumores histiocíticos.

\section{Relato de caso}

fêmea canina, poodle, de 8 anos foi encaminhada para atendimento devido histórico de epistaxe recorrente e dificuldade respiratória. Posteriormente foi submetida ao exame clínico de rotina, onde constatou-se uma lesão ulcerada no plano nasal, com evolução de aproximadamente seis meses. $\mathrm{O}$ nódulo macroscopicamente media $0,5 \mathrm{~cm}$, era circunscrito e arredondado, obstruía parte da narina e apresentava-se firme, aderido a mucosa drenando secreção serosanguinolenta, ademais essa foi a queixa principal apresentada na clínica.

O material foi fixado em formol tamponado a $10 \%$ e após fixação foi encaminhado para processamento histopatológico de rotina. Após emblocado em parafina, foi cortado e corado com Hematoxilina e Eosina (HE), e posteriormente lido.

No exame histopatológico observou-se um tumor não encapsulado e densamente proliferado, que envolvia parte da camada epidérmica e a derme, são fibroblastos com presença de grupos de núcleos 
alongados. Esses fibroblastos variam de alongados a arredondados, núcleos variáveis de tamanhos exorbitantes com cromatina frouxa e por vezes mais de um nucléolo. Citoplasma escasso e basofílico.

Na derme superficial e profunda nota-se as múltiplas camadas de histiócitos com atipia celular, núcleo de tamanho variável, com acentuada cariomegalia e citoplasma abundante e eosinofílico, nucléolos evidentes, por vezes a cromatina condensada impossibilita a visualização dos nucléolos, entremeando formações fibrosas densas (Figura 1A). Formação de espaços pseudovasculares preenchidos por sangue que se entremeiam as células histiocíticas de forma não uniforme e densa, distribuídos aleatoriamente (Figura 1B). O infiltrado inflamatório presente na amostra é essencialmente linfoplasmocitário, com raros neutrófilos e eosinófilos, estes entremeiam as células pleomórficas. Atividade mitótica baixa, variando de uma a duas mitoses por campo de maior aumento (objetiva de 40x).

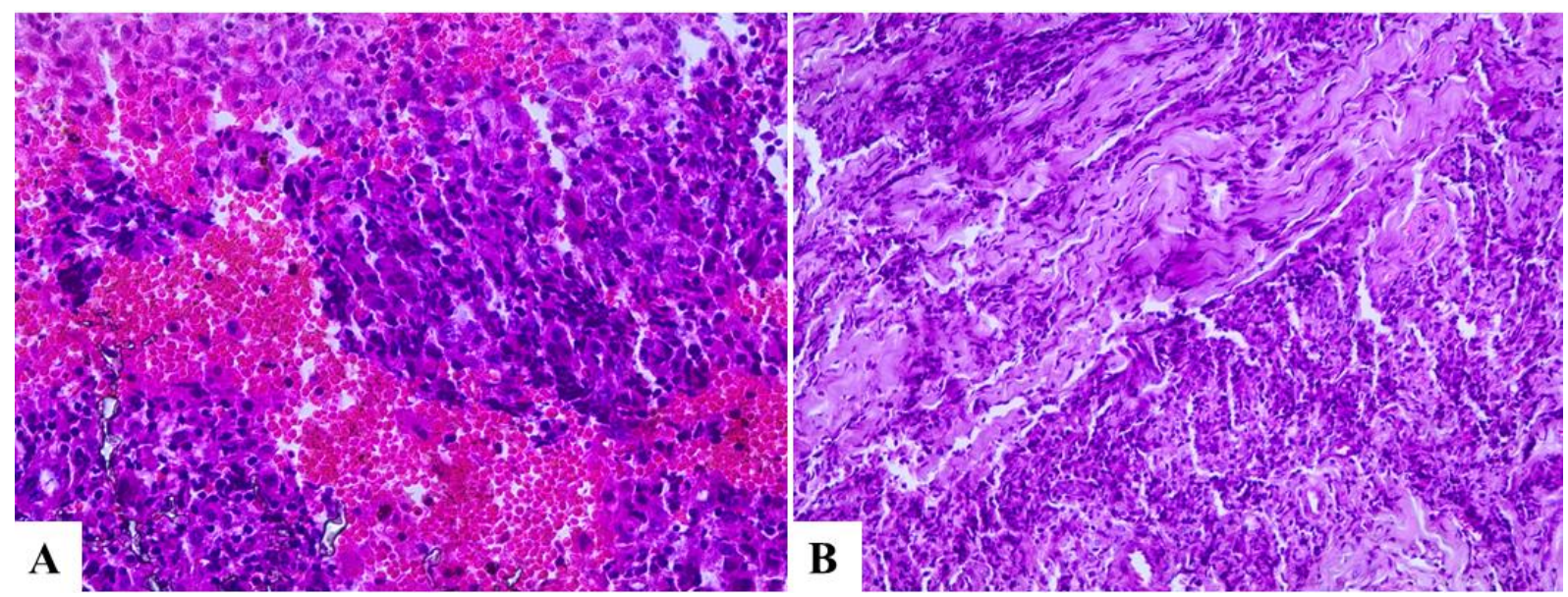

Figura 1. Fragmento de plano nasal de cão, poodle, histiocitoma fibroso maligno. (A). Derme superficial- nota-se espaços pseudovasculares preenchidos por sangue que se entremeiam as células histiocíticas de forma não uniforme e densa, distribuídos aleatoriamente. Hematoxilina e Eosina (HE). (Obj de 40x). (B). Derme profunda- evidenciam-se múltiplas camadas de histiócitos com atipia celular, núcleo de tamanho variável, com acentuada cariomegalia e citoplasma abundante e eosinofílico, nucléolos evidentes, por vezes a cromatina condensada impossibilita a visualização dos nucléolos, entremeando formações fibrosas densas. (Obj de 20x).

Os espécimes emblocados em parafina foram submetidos a cortes de $4 \mu \mathrm{m}$ de espessura. Após desparafinização em xilol, hidratação em concentrações decrescentes de etanol e lavagem em água deionizada, os cortes histológicos foram submetidos à recuperação antigênica em solução de citrato $10 \mathrm{mM}$ (pH 6.0) em câmara pressurizada (Pascal ${ }^{\circledR}$, Dako). Após a recuperação antigênica foi realizado o bloqueio da peroxidase endógena com peróxido de hidrogênio pronto para uso (Peroxide Block ${ }^{\circledR}$, Cell Marque, 925B-09) e bloqueio de proteínas inespecíficas (Dako ® Protein Block Serum-Free, X0909).

O anticorpo primário utilizado foi o HLA-DR, policlonal (Spring, E 4520) na diluição de 1:50, incubado por 18 horas a 4oC. Posteriormente, as lâminas foram lavadas em solução tampão TRIS pH 7,4 e, em seguida foram incubadas com sistema de detecção por polímero (HISTOFINE ${ }^{\circledR}$, NICHIREI, 414154F) de acordo com recomendações do fabricante. A imuno coloração foi obtida utilizando-se DAB (3,3' diaminobenzidinetetrachloride, Dako ${ }^{\circledR}$ ). Os cortes foram contra corados com Hematoxilina de Mayer e desidratados em concentrações crescentes de álcool e xilol. Em seguida, as lâminas foram montadas com resina e lamínula. Controles positivos e negativos foram adicionados a reação, a fim de atestar a fidelidade dos resultados.

As marcações foram positivas para o complexo de histocompatibilidade MHC II (HLA-DR), marcando intensamente as células neoplásicas proliferadas correspondentes aos histiócitos, consequentemente destacou os linfócitos presentes no infiltrado inflamatório, marcando positivamente com uma coloração marrom (Figura $2 \mathrm{~A} \mathrm{e} \mathrm{B}$ ) de forma difusa na amostra submetida ao exame de imuno-histoquímica. 


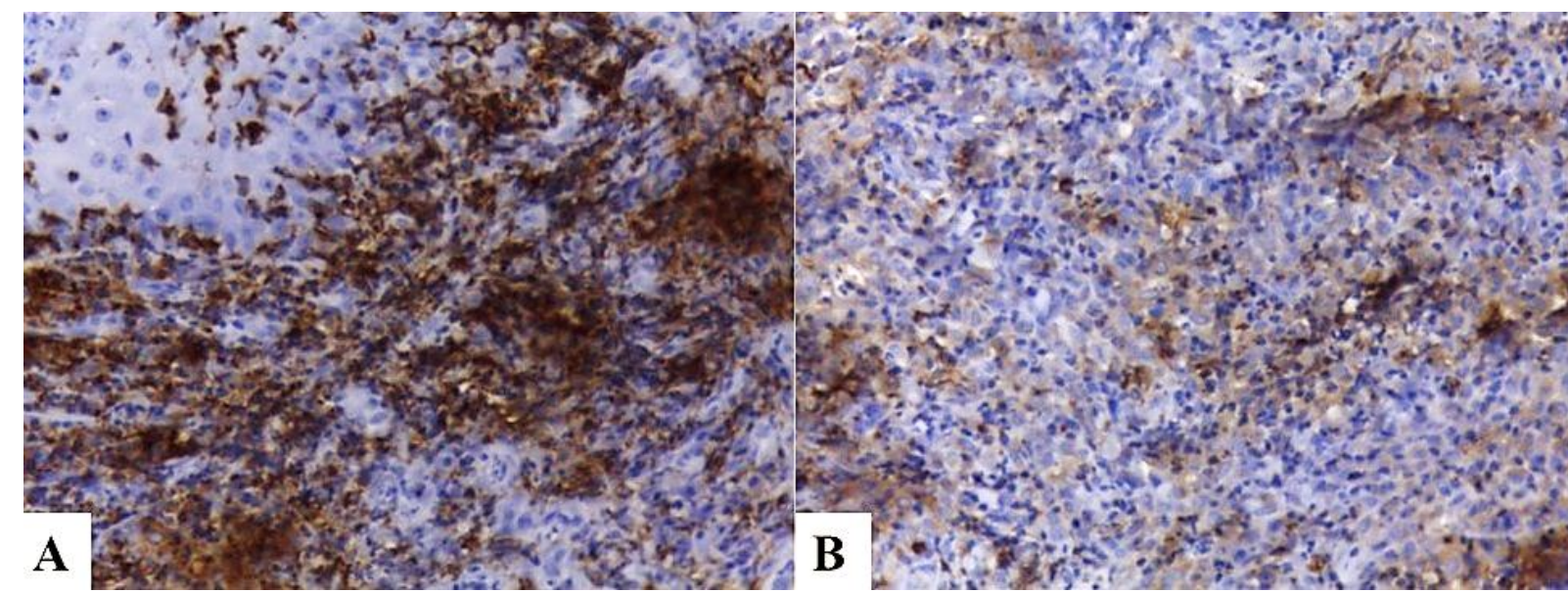

Figura 2. (A e B). Fragmento de plano nasal de cão, poodle, histiocitoma fibroso maligno. Aplicação de anticorpo HLA-DR, complexo de histocompatibilidade MHCII, com marcação fortemente positiva, de cor castanho, para células linfóides e histiocíticas que representam a área tumoral. (Obj de 40x).

\section{Discussão}

Os histiocitomas geralmente apresentam-se como nódulos isolados em regiões cutâneas, como no presente relato, havendo poucos casos sistêmicos descritos em animais até o momento, diferente das histiocitoses, que em alguns casos pode apresentar-se de forma sistêmica, acometendo pele e vísceras (Teixeira et al., 2011). De modo geral os histiocitomas podem regredir naturalmente, de maneira ainda não esclarecida, porém entende-se que possa estar relacionada com a migração das células para os linfonodos que são responsáveis pela drenagem da pele (Meuten, 2016), nesse caso relatado foi realizada a retirada do neoplasma e não houve recidivas a curto prazo.

O histiocitoma fibroso é um importante diferencial de outros tumores de pele histiocíticos bem como os outros tumores de células redondas mais comumente diagnosticados, como os mastocitomas e sarcomas, especialmente os sarcomas podem ser confundidos com histiocitomas fibrosos malignos. Diferencia-se especialmente o subtipo de histiocitoma fibroso de tumores epiteliais como carcinomas anaplásicos, uma vez que as células tumorais são bem diferenciadas e o diagnóstico assertivo depende da percepção do patologista quando aos diferenciais aplicáveis, levando em consideração o uso do histopatológico (Goldschmidt \& Hendrick, 2002; Moore, 2014), nesses casos o auxílio da imunohistoquímica pode ser aplicado (Paździor-Czapula et al., 2015; Stilwell \& Rissi, 2018).

Os aspectos da derme coincidem com o histicitoma fibroso aneurismal em humanos, que consiste na proliferação de múltiplos espaços vasculares com células pouco diferenciadas (Nabatanzi et al., 2019) já de acordo com a classificação veterinária se enquadra no histiocitoma fibroso, pela proliferação de células histiocíticas pleomórficas associada a fibroblastos, e presença de canais vasculares entremeando as células neoplásicas da derme (Meuten, 2016; Moore, 2014).

Não foram observadas possíveis metástases que são consideradas menos frequentes em histiocitomas (Lew et al., 2003; Moore, 2014; Teixeira et al., 2011). Todavia, existe a possibilidade de sub-diagnóstico uma vez que a classificação de histiocitomas, baseada na classificação humana é em parte aceita por patologistas veterinários e alguns subtipos não são aplicáveis. Comumente encontra-se relatos de tumores não classificados de acordo com as suas características morfológicas/histopatológicas em animais.

Visto que o histopatológico pode não ser totalmente assertivo quanto ao subtipo tumoral histiocítico é importante avaliar a necessidade de outros meios diagnósticos auxiliares como a imuno-histoquímica. No presente caso foram utilizados marcadores de complexo de histocompatibilidade MHC II, fenótipo compatível com células linfoides e histiocíticas, marcando de forma moderada a intensa em regiões de derme superficial e profunda, consequentemente comprovando se tratar de uma proliferação histiocítica neoplásica (Daleck et al., 2016; Kamstock et al., 2011; Pazdzior-Czapula et al., 2019; Webster et al., 2011). Para diferenciar histiocitoma fibroso maligno de sarcomas histiocíticos hemofagocíticos deve-se fazer o anticorpo CD11, uma vez que este tem capacidade de marcar células que vem da polpa vermelha esplênica (Meuten, 2016). 
Para o prognóstico e tratamento é de suma importância uma subclassificação desses tumores histiocíticos, uma vez que alguns são considerados mais agressivos e recidivantes do que outros (Moore, 2014; Pires et al., 2013). Existem poucos relatos de histocitomas fibrosos, nesses relatos os neoplasmas apresentam-se como tumores pouco infiltrativos, porém não encapsulados e de prognóstico reservado com tratamento efetivo cirúrgico e poucos dados relativos a tratamentos, metástases e recidivas uma vez que estes não estão bem esclarecidos (Meuten, 2016; Moore, 2014).

Os histiocitomas são comuns, os fibrosos e malignos, menos comumente diagnosticados. Somente o diagnóstico histopatológico é capaz de diferenciar os tipos histiocíticos e suas subclassificações, muitas vezes faz-se necessário a complementação com o exame imuno-histoquímico para diferenciar de padrões histopatológicos presentes em outras neoplasias.

\section{Conflito de Interesse}

Os autores declaram não existir conflito de interesse.

\section{Agradecimentos}

Os autores desse artigo agradecem aos colaboradores do Hospital Veterinário da Paraíba-UFPB pela contribuição indireta a este manuscrito.

\section{Referências}

Affolter, V. K., \& Moore, P. F. (2006). Feline progressive histiocytosis. Veterinary Pathology, 43(5), 646-655. DOI: https://doi.org/10.1354/vp.43-5-646

Daleck, C. R., Fonseca, C. S., \& Canola, J. C. (2016). Oncologia em cães e gatos. Roca.

Doyle, L. A., \& Fletcher, C. D. M. (2013). Metastasizing "benign" cutaneous fibrous histiocytoma: a clinicopathologic analysis of 16 cases. The American Journal of Surgical Pathology, 37(4), 484495. DOI: https://doi.org/10.1097/PAS.0b013e31827070d4

Goldschmidt, M. H, \& Hendrick, M. J. (2002). Tumors of the skin and soft tissues. In D J Meuten (Ed.), Tumors in Domestic Animals (pp. 45-117). Iowa State Press. DOI: https://doi.org/10.1002/9780470376928.ch2

Goldschmidt, Michael H, \& Goldschmidt, K. H. (2017). Epithelial and melanocytic tumors of the skin. In D J Meuten (Ed.), Tumors in Domestic Animals (pp. 88-141). John Wiley \& Sons Inc. DOI: https://doi.org/10.1002/9781119181200.ch4

Gross, T. L., Ihrke, P. J., Walder, E. J., \& Affolter, V. K. (2008). Skin diseases of the dog and cat: clinical and histopathologic diagnosis. John Wiley \& Sons.

Kamstock, D. A., Ehrhart, E. J., Getzy, D. M., Bacon, N. J., Rassnick, K. M., Moroff, S. D., Liu, S. M., Straw, R. C., McKnight, C. A., \& Amorim, R. L. (2011). Recommended guidelines for submission, trimming, margin evaluation, and reporting of tumor biopsy specimens in veterinary surgical pathology. Veterinary Pathology, 48(1), 19-31. DOI: https://doi.org/10.1177/0300985810389316

Lew, W., Lim, H. S., \& Kim, Y. C. (2003). Cutaneous metastatic malignant fibrous histiocytoma. Journal of the American Academy of Dermatology, 48(2), S39-S40. DOI: https://doi.org/10.1067/mjd.2003.113

Meuten, D. J. (2016). Tumors in domestic animals. John Wiley \& Sons.

Moore, P. F. (2014). A review of histiocytic diseases of dogs and cats. Veterinary Pathology, 51(1), 167-184. DOI: https://doi.org/10.1177/0300985813510413

Nabatanzi, A., Male, M., Qu, X., Li, Y., Meng, X., Di, W., \& Huang, C. (2019). Aneurysmal Fibrous Histiocytoma: Clinicopathology Analysis of 30 Cases of a Rare Variant of Cutaneous Fibrohistiocytoma. Current Medical Science, 39(1), 134-137. DOI: https://doi.org/10.1007/s11596019-2011-9

Pazdzior-Czapula, K., Mikiewicz, M., Gesek, M., Zwolinski, C., \& Otrocka-Domagala, I. (2019). Diagnostic immunohistochemistry for canine cutaneous round cell tumours-retrospective analysis 
of 60 cases. Folia Histochemica et Cytobiologica, 57(3), 146-154. DOI: https://doi.org/10.5603/fhc.a2019.0016

Paździor-Czapula, K., Rotkiewicz, T., Otrocka-Domagała, I., Gesek, M., \& Śmiech, A. (2015). Morphology and immunophenotype of canine cutaneous histiocytic tumours with particular emphasis on diagnostic application. Veterinary Research Communications, 39(1), 7-17. DOI: https://doi.org/10.1007/s11259-014-9622-1

Pinto da Cunha, N., Ghisleni, G., Scarampella, F., Fabbrini, F., Sforna, M., Cornegliani, L., Caniatti, M., Avallone, G., Moore, P., \& Roccabianca, P. (2014). Cytologic and immunocytochemical characterization of feline progressive histiocytosis. Veterinary Cinical Pathologylinical, 43(3), 428436. DOI: https://doi.org/10.1111/vcp.12152

Pires, I., Alves, A., Queiroga, F. L., Silva, F., \& Lopes, C. (2013). Regression of canine cutaneous histiocytoma: reduced proliferation or increased apoptosis? Anticancer Research, 33(4), 1397-1400.

Stilwell, J. M., \& Rissi, D. R. (2018). Immunohistochemical Labeling of Multiple Myeloma Oncogene 1/Interferon Regulatory Factor 4 (MUM1/IRF-4) in Canine Cutaneous Histiocytoma. Veterinary Pathology, 55(4), 517-520. DOI: https://doi.org/10.1177/0300985818759770

Teixeira, L., Ferreira, K. C. R. S., Oliveira, L. O., Kroetz, R. M., Oliveira, R. T., Sonne, L., \& Pavarini, S. P. (2011). Histiocitose maligna sistêmica em um cão-Relato de caso. Medvep - Revista Científica de Medicina Veterinária - Pequenos Animais e Animais de Estimação., 9(28), 97-101.

Webster, J. D., Dennis, M. M., Dervisis, N., Heller, J., Bacon, N. J., Bergman, P. J., Bienzle, D., Cassali, G., Castagnaro, M., \& Cullen, J. (2011). American College of Veterinary Pathologists' Oncology Committee et al: Recommended guidelines for the conduct and evaluation of prognostic studies in veterinary oncology. Veterinary Pathology, 48, 7-18. DOI: https://doi.org/10.1177/0300985810377187

\section{Histórico:}

Recebido: 11 de setembro de 2020 Aprovado: 19 de outubro de 2020 Disponível online: 9 de dezembro de 2020.
Licenciamento: Este artigoé publicado na modalidade Acesso Aberto sob a licença Creative Commons Atribuição 4.0 (CC-BY 4.0), a qual permite uso irrestrito, distribuição, reprodução em qualquer meio, desde que $\mathrm{o}$ autor e a fonte sejam devidamente creditados. 